\title{
La régulation de l'emploi dans les hypermarchés français.
}

Stratégies de mobilisation de la main-d'œuvre et rapports à l'activité aux caisses

Employment regulation in French hypermarkets. Strategies to mobilize the workforce and cashiers' relationship to work

\section{Mathias Waelli et Philippe Fache}

\section{OpenEdition \\ Journals}

Édition électronique

URL : https://journals.openedition.org/travailemploi/6128

DOI : 10.4000/travailemploi.6128

ISSN : 1775-416X

Éditeur

DARES - Ministère du Travail

\section{Édition imprimée}

Date de publication : 14 décembre 2013

Pagination : $35-47$

ISSN : 0224-4365

Référence électronique

Mathias Waelli et Philippe Fache, «La régulation de l'emploi dans les hypermarchés français. », Travail et Emploi [En ligne], 136 | octobre-décembre 2013, mis en ligne le 01 octobre 2015, consulté le 21 septembre 2021. URL : http://journals.openedition.org/travailemploi/6128 ; DOI : https://doi.org/ 10.4000/travailemploi.6128 


\title{
La régulation de l'emploi dans les hypermarchés français. Stratégies de mobilisation de la main- d'œuvre et rapports à l'activité aux caisses ${ }^{(*)}$
}

\author{
Mathias Waelli(**), Philippe Fache ${ }^{(* * *)}$
}

Les régimes de mobilisation de la main-d'œuvre dans la grande distribution ont longtemps reposé sur la promotion interne et sur la fragmentation du marché de l'emploi. Alors que des travaux mettent déjà en lumière le déclin de la logique méritocratique, nos observations, réalisées dans le courant des années 2000, soulignent une érosion des ressorts des politiques de précarisation des contrats de travail en caisse pour mobiliser la main-d'œuvre. En partant de ce constat, qui est matérialisé par une augmentation du turnover et de l'absentéisme à partir du début des années 2000, cet article explore la distance séparant les perspectives réelles en caisse des attentes des employés qui résultent d'arbitrages entre des activités (salariales, domestiques, familiales, etc.) situées dans plusieurs sphères de vie. Ilmet en lumière une tension générée par l'émancipation des caissiers vis-à-vis des catégories traditionnelles - aspiration au CDI à temps plein - qui constituent encore le socle de la relation salariale.

Les régimes de mobilisation de la main-d'œuvre dans la grande distribution française ont longtemps reposé, et reposent encore en partie, sur la promotion interne pour les employés de rayon non qualifiés, et sur la fragmentation du marché de l'emploi pour les employés de caisses. Dans ses travaux récents (2012a), Sophie BERNARD souligne déjà le déclin de la logique méritocratique. Aujourd'hui, de plus en plus de distributeurs recourent à de jeunes diplômés pour tenir les postes d'encadrement. Nos observations dans le secteur révèlent, quant à elles, une érosion des ressorts de la régulation de l'emploi par la précarisation des contrats. L'article part de ce constat, établi en région parisienne, pour étudier les rapports des caissiers à l'emploi et à «l'activité»; autrement dit, il s'intéresse aux arbitrages effectués par les caissiers entre les activités situées dans différentes sphères de vie, en écho à la notion d' «activité vitale» de Chantal NicoleDranCOURT (2004).

L'étude, réalisée par immersion (voir encadré 1), met l'accent sur la variété des pratiques et des représentations. En ce sens, elle s'inscrit dans la lignée de travaux antérieurs (BEAU, 2004 ; Le CORRE, 1991,

(*) Les auteurs tiennent à remercier les rapporteurs anonymes et l'équipe de Travail et emploi pour leurs remarques et leur relecture attentive.

(**) Institut du management, École des hautes études en santé publique (EHESP), EA Management des organisations de santé (MOS); Mathias.waelli@ehesp.fr

(***) École supérieure de commerce et de marketing ICD -Paris/Lara (Laboratoire de recherche appliquée), Université Paris 13 : pfache@groupe-igs.fr
2001) en observant une grande hétérogénéité à la fois des formes de gestion sociale, des collectifs et des parcours inhérents au secteur de la distribution.

Concernant toujours le profil des salariés et le rapport à l'activité, la période qui s'étend des années 2001 à 2006, pendant laquelle nous avons recueilli nos données de terrain, se présente comme un moment-clé dans la distribution. Les distributeurs y emploient un nombre croissant d'étudiants en caisse, population dont Sophie BERnARD et Flore ChAPPAZ (2007) pointent à la fois le poids dans les collectifs et le faible degré de dépendance à l'emploi. Durant cette période, la proportion des étudiants à temps partiel, initialement marginale, tend à rattraper celle des caissières à temps presque complet. Le phénomène, qui se matérialise dans les magasins enquêtés par l'augmentation en cinq ans de $10 \%$ à $50 \%$ de la part des emplois d'étudiants en caisse, est le produit d'une évolution à la fois des marchés locaux d'emploi et des modalités d'organisation du travail. En effet, même si, contrairement aux idées reçues, la massification scolaire n'a pas produit d'accroissement significatif du nombre de salariés étudiants en France (BERNARD, ChaPpAZ, 2007), elle a conduit à une transformation des marchés locaux de l'emploi (PInTo, 2007). C'est le cas en particulier dans les banlieues où sont situées les grandes surfaces commerciales. Dans le même temps, le passage, à la fin des années 1990, d'un modèle de croissance «expansive» à un modèle de croissance «intensive» (MoATI, 2001) marqué par la concurrence a conduit les magasins à adopter des amplitudes horaires de plus en plus étendues, favorisant la multiplication des contrats étudiants (pour une durée de douze à vingt heures 
par semaine, avec prise de poste en fin de journées et le samedi). Ainsi, à la fin de la période observée, les distributeurs ont développé des politiques orientées spécifiquement vers ces populations (nous renvoyons ici au «concept étudiant» de Carrefour). Concomitamment, on a alors assisté au relatif déclin de l'efficacité des modalités de mobilisation de la main-d'œuvre par le sous-emploi. Le phénomène est souligné, dans quatre des magasins enquêtés, par une forte progression des contrats étudiants en caisse : entre 1998 et 2005 , ils sont passés d'une moyenne de $9 \%$ à $45 \%{ }^{(1)}$. Parallèlement, dans l'ensemble de la grande distribution, l'absentéisme a augmenté de manière importante et frôle en moyenne les $9 \%$ (2) tandis que les taux de turnover ont doublé et atteignent souvent $100 \%$ aux caisses des hypermarchés. Le phénomène de l'absentéisme fait dès lors durablement figure de fléau. En témoigne l'initiative prise par l'enseigne Carrefour en 2006 qui, confrontée à un taux record de $9 \%$, s'engage dans une politique volontariste de lutte couronnée en 2010 par la mise en place d'un système d'octroi de primes récompensant les efforts, magasin par magasin.

Dans ce contexte particulier, l'article interroge la tension opposant des modalités déclinantes de mobilisation de la main-d'œuvre à une évolution du rapport des caissiers à l'emploi et à l'activité. Du côté des salariés, il montre que l'on assiste à l'apparition de multiples compromis, fondant de nouvelles formes de rapport à l'emploi et à l'activité et de plus en plus éloignés de la norme de l'emploi stable à temps plein.

Dans un premier temps, l'article présente certaines spécificités de l'évolution du travail et de l'emploi en caisse, qui conduisent à le stigmatiser et à masquer la variété des profils dans les collectifs. Il pointe ensuite une modification des régimes de mobilisation des salariés, révélatrice de profondes mutations des représentations. Enfin, grâce à une typologie des rapports à l'activité et à l'emploi, il révèle tout à la fois la diversité des compromis et le creusement généralisé de l'écart entre les attentes des salariés et les conditions de travail et d'emploi en caisse ${ }^{(3)}$.

(1) Pour ces quatre magasins, nous avons obtenu les détails des bilans sociaux. Par ailleurs, les taux de turnover pour l'enseigne et l'ensemble de la branche (diffusés par la Fédération des entreprises du commerce et de la distribution) ne distinguent pas les caisses des autres départements des magasins, bien qu'ils obéissent à des logiques d'emploi spécifiques. En général, les taux de turnover y sont deux à trois fois plus élevés et l'emploi à temps partiel y constitue la norme.

(2) Source: Fédération du commerce et de la distribution, Rapport de branche 2006.

(3) Cette insatisfaction s'est partiellement exprimée dans les revendications de 2008, dont Marlène Benquet (2011) a démonté les ressorts autour d'une triple précarisation de l'emploi en caisse, à la fois économique, organisationnelle et «projectionnelle».

\section{Encadré 1 Méthode d'enquête}

Cette étude repose sur l'articulation de trois éclairages complémentaires: observation participante dans la durée, entretiens approfondis et analyse de données quantitatives de seconde main.

Les observations participantes ont été menées à couvert entre avril 2001 et janvier 2006: tout d'abord avec un contrat étudiant classique de quinze heures par semaine (identifié par les collègues comme tel), puis grâce à une convention Cifre ${ }^{(1)}$; l'enquêteur était alors identifié par les managers comme un stagiaire et par les collègues comme un étudiant en thèse réalisant une enquête sur les conditions de travail. II a donc partagé les conditions de travail (et parfois d'emploi) des caissiers et des employés commerciaux dans quatre hypermarchés d'une même enseigne, situés respectivement en Île-de-France (neuf mois), en région lilloise (six mois), aux alentours de Metz (six semaines) puis à Limoges (quinze jours), avant un retour d'un mois dans le premier magasin. Ces magasins étaient tous de grande taille (entre 10000 et 18000 mètres carrés de surface commerciale) avec des collectifs de caisse comprenant entre 100 et 190 employés.

Pour saisir l'ensemble des comportements et des attentes des travailleurs, il a fallu sortir du cadre strict des observations en entreprise. Cette nécessité est renforcée en caisse car le degré d'identification au travail y est généralement faible. Par ailleurs, l'enquêteur gagne à privilégier le contexte de l'entretien en fonction de son objet d'étude. Ainsi, après avoir travaillé quelque temps auprès des salariés, l'enquêteur a réussi à gagner la confiance de certains d'entre eux, ce qui lui a permis de réaliser des entretiens approfondis $(n=60)$ dans des conditions propices à l'observation de l'articulation entre le travail et le hors-travail : ils se sont principalement déroulés dans des lieux publics (cafés, restaurants) où les salariés avaient leurs habitudes ou, mieux encore, s'agissant des mères de familles, dans leur foyer. Dans certains cas, les autres membres de la famille ont même pris part à la discussion de sorte que l'enquêteur a pu mieux saisir l'ensemble des contraintes domestiques conditionnant leurs attentes.

Enfin, l'enquête repose aussi sur l'analyse de données statistiques de seconde main, principalement celles présentées dans les bilans sociaux de l'entreprise, notamment les taux de turnover et d'absentéisme magasin par magasin, et celles proposées par les rapports annuels de branche de la Fédération des entreprises du commerce et de la distribution (FCD) pour la période étudiée. Ces données ont parfois infléchi le cours des observations et ont conduit l'enquêteur à réaliser des terrains comparatifs en Île-de-France et en province. Les chiffres contribuent à rendre compte de l'ampleur et de l'inscription dans la durée des processus observés ponctuellement sur le terrain.

(1) Conventions industrielles de formation par la recherche. 


\section{L'emploi en caisse, un symbole de pénibilité et de précarité qui fait écran à la variété des pratiques}

En matière de mobilisation de la main-d'œuvre, la grande distribution française se présente à la fois comme un univers singulier et un exemple significatif. En effet, dans un contexte où l'organisation du travail n'a pas été remise en cause par la conjoncture économique, contrairement à nombre d'autres secteurs d'activité, les politiques de ressources humaines se sont concentrées sur le recours à la précarisation des contrats pour mobiliser la main-d'œuvre en caisse. La généralisation de ces politiques a été telle que «la caissière» est devenue une victime emblématique des logiques induites par la fragmentation de l'emploi (ANGElofF, 2000; ApPAY, 2005; DuRAND, 2004); elle offre une sorte d'image d'Épinal univoque qui assimile temps partiel à précarité et, ce faisant, peut masquer la variété des compromis et des stratégies d'emploi des salariés.

\section{Une activité de travail dévalorisée}

L'évolution de l'organisation du travail s'inscrit dans un contexte particulier. Contrairement au modèle de production fordiste qui lui est associé (MoATI, 2001), la grande distribution n'a en effet pas connu de crise. En France, le modèle a pourtant atteint certaines limites, en partie sous la contrainte de dispositifs juridiques comme la loi Royer en 1972 puis la loi Raffarin en 1996, alors que la croissance expansive se poursuivait dans les pays émergents. Cependant, depuis l'ouverture des premiers hypermarchés, les fondements du modèle du discompte (discount) n'ont guère été remis en cause par le marché. Ces dernières années, comme en témoigne le succès du hard discount, ils ont même été confortés par des clients inquiets de la perte de leur pouvoir d'achat. Ainsi, dans un secteur peu menacé, les distributeurs n'ont pas eu à reconsidérer l'organisation et la nature du travail, a fortiori pour les caissiers. Les évolutions les plus visibles, comme le changement de dénomination des employés (de «caissiers», ils sont devenus «hôtes de caisse») ou la mise en scène des métiers dans la surface commerciale, consistent essentiellement en des messages à destination des clients. Elles ne visent pas à mobiliser les salariés autour du contenu du travail lui-même, travail dont le caractère à la fois peu attractif et transitoire est au contraire souligné dès l'embauche par les managers eux-mêmes. Le discours de ce directeur de magasin est, de ce point de vue, révélateur :

"Il ne faut pas se leurrer, hôtesse de caisse, ce n'est pas un travail épanouissant. C'est très bien pour des jeunes étudiants qui font leurs premiers pas dans le monde de l'entreprise, pour des mères de famille qui cherchent un petit appoint, mais ce n'est pas un lieu pour faire carrière. D'ailleurs, le manager des caisses les avertit toujours à l'embauche. Et puis, comme je le dis toujours, les employés, c'est comme les clients, ça ne sert à rien de leur mentir sur la marchandise, sinon, on risque de casser la relation de confiance et la confiance, c'est essentiel. »

Certes, l'activité de travail en caisse n'a cessé d'évoluer depuis cinquante ans. Sophie PrunIER Poulmaire (2000) a ainsi montré que l'introduction de la technologie, et du scanner en particulier, a renforcé son intensification aux caisses traditionnelles. Plus récemment, la mise en place de caisses automatiques a transformé la relation au client et induit de nouvelles contraintes physiques $\left(\mathrm{B}_{\mathrm{A}}\right.$, VignON, 2013; Bernard, 2012b). Cependant, à l'inverse de ce que l'on a pu observer parfois au sein du monde industriel, ces changements n'ont jamais fait l'objet d'une politique de valorisation de l'activité de travail. Au contraire même, le travail dans la grande distribution souffre d'une image de plus en plus dégradée, notamment en raison d'études épidémiologiques qui la classent régulièrement parmi les mauvais élèves en matière de santé au travail. Dès 2004, face aux interrogations suscitées par l'augmentation du turnover comme de l'absentéisme et par la croissance significative du nombre de maladies professionnelles, les employeurs et les instances de représentation du personnel se sont accordés sur la nécessité d'établir un diagnostic concernant les répercussions de l'organisation du travail sur la santé. Les résultats de l'étude Ergodistrib (Bortel et al., 2007) puis, plus récemment, ceux diffusés par 1'Association des centres médicaux de soins (ACMS) en 2010 (CHANeY et al., 2011) révèlent que $85 \%$ des salariés considèrent leur travail fatigant physiquement. Ils mettent l'accent sur les conditions physiques de travail et les tensions liées à l'organisation du travail, au croisement des injonctions hiérarchiques et des pressions de la relation avec les clients; ils insistent également sur le stress dû aux incertitudes sur les horaires de travail qui renforcent les difficultés d'articulation entre vie privée et vie professionnelle. Paradoxalement, la pénibilité objective justifie de la part des employeurs - qui ont manifesté un intérêt pour l'établissement d'un diagnostic sur l'état de santé des salariés - le recours plus important au temps partiel afin de limiter l'exposition de leurs employés aux risques professionnels. Du côté des salariés, le temps partiel constitue aussi parfois une stratégie pour pouvoir tenir et faire face à la pénibilité des conditions de travail. Cependant, ces tendances peuvent alimenter simultanément d'autres formes de souffrance liées cette fois aux conditions d'emploi (dépassements d'horaires, revenus insuffisants, etc.).

\section{Un constat généralisé d'une dégradation des conditions d'emploi}

Des années 1990 jusqu'au début des années 2000, de nombreux travaux ont montré que la mobilisation de la main-d'œuvre en caisse reposait beaucoup sur la précarisation (Bouffartigue, Pendariès, 1994; 
LaLlement, 1998, etc.) sous-tendue par la logique du sous-emploi CATTANÉo (1997).

Les chiffres de la Fédération des entreprises du commerce et de la distribution relatifs à la période étudiée (4) confortent ce constat : contrairement aux idées reçues, la grande distribution emploie alors, tous métiers confondus, une forte proportion de salariés en contrat à durée indéterminée $(\mathrm{CDI})^{(5)}$, comparable à celle des caisses dans les magasins pour lesquels nous avons eu accès aux bilans sociaux. La grande distribution connaît dans le même temps un turnover très élevé $(57 \%$ en 2005) révélant la faible attractivité des contrats proposés.

Par contre, dans les hypermarchés et les supermarchés, la force de travail est constituée d'une part significative de temps partiels. En 2006, le taux atteint ainsi $46 \%$ dans les supermarchés et $55 \%$ en hypermarchés ${ }^{(6)}$. Cette tendance est plus marquée pour les caissiers, qui forment la catégorie professionnelle la plus concernée par le temps partiel (7), avec des pics pouvant atteindre dans certains magasins jusqu'à $98 \%$ des contrats.

\section{Sur le terrain, une grande variété de pratiques}

Toutefois, se limiter à une définition trop étroitement contractuelle de la précarité (absence de CDI à temps plein) fait courir le risque d'assimiler le temps partiel au sous-emploi. Or en caisse, tous les salariés à temps partiel ne sont pas des «précaires». Dans leurs travaux ethnographiques, Isabelle GARABUAU (1997) - qui ne traite pas, par ailleurs, la question du rapport à l'emploi - puis Sophie BERNARD et Flore ChAPPAZ (2007) ont relevé l'importance de la présence étudiante au sein des collectifs. Nos propres observations soulignent en outre, qu'en dehors des travailleurs étudiants qui, bénéficiant de soutiens matériels de leur famille, n'ont pas toujours besoin d'un temps plein, de nombreux caissiers effectuent des arbitrages professionnels ni au profit de l'emploi le plus stable, ni en faveur de la quotité de temps de travail la plus élevée (voir encadré 2).

(4) Dans l'ensemble, ces tendances persistent encore jusqu'à aujourd'hui.

(5) La grande distribution reste sur ce point dans les normes avec un chiffre stable de $87 \%$ de CDI sur la période 2001-2005 (source : FCD) contre $86,4 \%$ pour l'ensemble du monde du travail (source: Insee, Enquête Emploi 2006).

(6) Source: Contrats d'étude prospective. Commerce de détail et de gros à prédominance alimentaire. Rapport final validé lors du comité de pilotage du 3 avril 2008, Paris, Geste, Credoc, 2008.

(7) En 2004-2005, $45 \%$ des hommes sont à temps partiel et $60 \%$ des femmes (source : Geste, Credoc, 2008).

\section{Encadré 2}

\section{Martine, entre précarité de l'emploi et maîtrise du temps à soi}

En caisse depuis vingt-et-un ans dans le même magasin de la région lilloise, Martine élève seule un fils de 17 ans. Elle dit souvent de lui qu'il exige de «moins en moins de présence à la maison» et de "plus en plus d'apports financiers". La semaine précédant le début de l'observation en magasin, elle a pourtant refusé la proposition qui lui était faite par son manager d'un contrat de travail de trente heures hebdomadaires au lieu des vingt qu'elle effectuait jusqu'alors. Elle a préféré une solution qui consiste à effectuer une base de vingt heures de travail par semaine complétée par d'éventuelles heures supplémentaires. C'est «moins assuré», dit-elle, "mais plus souple». En effet, les contrats de vingt heures présentent une moins grande variabilité horaire qui facilite l'articulation de différentes activités, professionnelles ou non. "Je peux ainsi mieux gérer mon temps ", temps consacré à un second emploi, moins formel contractuellement et moins strict sur la disponibilité. "Je fais des ménages en complément [...], j'aime bien ça, je suis seule, je me sens libre. J'ai personne sur mon dos. Et puis les ménages c'est pas comme en caisse, je peux m'arranger plus facilement pour venir un autre jour. " Le temps ainsi libéré est également consacré depuis peu à une nouvelle relation amoureuse. Aussi, la perspective d'un contrat de trente heures, qui lui assurerait des rentrées d'argent régulières, ne l'intéresse guère. Et Martine ne surprend personne lorsqu'elle confie ses motivations en salle de pause : d'autres collègues ont déjà fait le même choix.

Plusieurs facteurs témoignent encore de l'ambivalence des aspirations à un statut plus stable, à commencer par le succès mitigé d'une mesure mise en place pour répondre aux mouvements sociaux de $2008^{(8)}$. Il s'agit alors d'en "finir avec la précarité». Pour atteindre cet objectif et tenter de mettre un terme au conflit, de nombreux magasins ont proposé de transformer les contrats à temps partiel des caissiers en contrats à temps complet; les heures complémentaires devaient alors être effectuées en rayon. Selon les témoignages de délégués syndicaux et de directeurs de magasin concernés par cette mesure, il semble qu'elle n'ait pas eu l'attractivité attendue auprès du personnel de caisse : selon les magasins, seulement 10 à $15 \%$ environ des effectifs à temps partiel ont saisi cette «opportunité». Les syndicats expliquent généralement ce peu d'engouement par les difficultés qu'ont rencontrées les personnels de caisse pour s'intégrer au sein des équipes de rayon et apprendre de nouvelles tâches qui auraient dû faire l'objet d'un effort de formation spécifique insuffi-

(8) Le $1^{\text {er }}$ février 2008, a eu lieu la première grève intersyndicale et interenseigne de la grande distribution française, un mouvement contre la «précarité» dont Marlène BENQUET (2011) propose une analyse détaillée. 
samment mis en œuvre par les distributeurs. Quelles que soient les explications, le phénomène montre bien, dans l'ensemble, qu'à l'image de Martine, les seules dimensions statutaires et salariales ne peuvent répondre à tous les besoins des salariés. La critique syndicale met l'accent sur l'importance du contenu de l'activité de travail alors que Martine souligne davantage son aspiration à plus d'autonomie. Ce sont alors les conditions de l'activité de travail et l'articulation avec le hors-travail qui, au moins au même titre que la durée contractuelle de travail hebdomadaire, sont sources de tensions pour les salariés. Ces arbitrages en faveur de solutions a priori plus fragiles placent parfois les caissiers en situation instable, de façon comparable au travail intérimaire qui peut être source de nouvelles dépendances cachées derrière le sentiment d'autonomie illusoire de gestion de son temps de travail (PIllon, 2008). Cependant, ils sont simultanément le signe d'une émancipation subjective vis-à-vis des normes qui fondent le rapport salarial français ${ }^{(9)}$, et soulignent ainsi l'enjeu croissant constitué par la maîtrise du temps dans des contextes de vie de plus en plus «fractionnés». Inscrits au coeur des logiques de «multi-activités» (CINGOLANI, 2012), ces multiples compromis laissent également entrevoir l'émergence de nouvelles formes de rapports à l'activité et à l'emploi en lien avec l'évolution des modalités de mobilisation de la main-d'œuvre (BuÉ et al., 2013).

\section{Une évolution des formes de mobilisation de la main-d'œuvre aux caisses significative de nouveaux compromis}

Ainsi, les compromis de plus en plus nombreux que font les salariés peuvent se saisir à travers le prisme des régimes de mobilisation de la main-d'œuvre. Si les distributeurs ont toujours pris appui sur la forme des contrats pour renforcer l'engagement des caissiers dans leur travail, ils doivent cependant s'adapter, selon les circonstances, à leurs stratégies d'emploi.

Nous avons pu observer, entre 2001 et 2006, qu'après une période relativement propice à la mobilisation de la main-d'œuvre dans un contexte de sous-emploi, les managers de caisses ont été contraints de réfléchir à d'autres politiques de ressources humaines pour augmenter l'attractivité du secteur.

\section{Logiques flexibles et segmentations en caisse}

Parallèlement à la montée du chômage, on a assisté depuis la fin des années 1980 à une forte

(9) Constituant donc encore une contrainte objective puisque l'emploi conditionne l'accès aux droits sociaux (LetABLIER, Nicole-Drancourt, 2007). fragmentation du marché de l'emploi aux caisses. Avec l'appui de l'État via les contrats d'insertion (Durand, 2004), les distributeurs ont diversifié les formes d'emploi, introduisant ainsi de nombreuses étapes dans la course présumée au CDI à temps complet. Dans le même temps, ils ont généralisé les embauches à temps partiel en caisses, qui ont fini par atteindre une proportion de $33 \%$ des contrats en 1992 dans les hypermarchés, tous départements confondus ${ }^{(10)}$. Les travaux de Bouffartigue et Pendariès (1994) ont montré combien ces mesures contribuaient à la fois à instaurer chez les salariés un sentiment d'évaluation permanente et une mise à disposition de leur temps, subordonnant toute activité de la sphère privée aux exigences du travail.

Le phénomène permet de mobiliser la maind'œuvre autour d'une activité de travail dont l'attrait a fortement diminué parmi les nouvelles générations de salariés. En effet, des années 1960 jusque dans les années 1980, les emplois de caisse ont peu ou prou correspondu aux profils arrivés alors en masse sur le marché du travail salarié (BATTAGLIOLA, 2004) : ceux de femmes peu qualifiées, principalement associées à la sphère domestique, que l'absence de légitimité dans le monde du travail contribuait à cantonner à des emplois de complément.

Depuis une vingtaine d'années, les nouvelles générations de salariées, ayant connu une forte progression de leur niveau de scolarisation et de réussite scolaire, sont poussées à développer de nouvelles ambitions professionnelles tout en continuant à prendre en charge des responsabilités familiales (DuBAR, 2000). Elles portent alors un regard plus critique sur une activité de travail peu qualifiée dans un secteur peu propice au développement des carrières. Le phénomène contribue à déporter l'attractivité du travail sur le salaire et la stabilité de l'emploi. Dans un contexte de marché de l'emploi défavorable, il concentre l'attention des jeunes employé(e)s sur les conditions d'emploi, c'est-à-dire sur l'opportunité de stabiliser leur situation et d'atteindre un niveau de salaire décent en faisant preuve d'une grande disponibilité pour l'entreprise.

Les nouvelles recrues ne sont pas les seules touchées par ce phénomène. Les anciennes, malgré la stabilité apparente de leur contrat, ne sont pas moins épargnées par la précarité. À l'occasion d'événements survenant dans la sphère privée (naissance, maladie d'un membre de la famille, etc.), elles sont régulièrement appelées à renégocier leur temps de présence en caisse et au foyer. Les managers prennent alors appui sur ce qui semble être un besoin de conciliation entre vie professionnelle et vie familiale exprimé par les salariées pour adapter leurs contrats (initialement des CDI à temps plein

(10) Elle n'était que de 6,2\% en 1972 et de $21 \%$ en 1982 (source : Insee Division Commerce). 
avec horaire fixe) aux normes de l'époque, avec, à la clé, temps partiel et horaires flexibles.

Les distributeurs expliquent généralement le recours à la flexibilité comme une réponse à la pénibilité, mais aussi, ainsi que l'indique le chef de caisses cité ci-dessous, comme une adaptation aux exigences incontournables du marché.

«De plus en plus, on a eu aussi un phénomène de fréquentation assez hétérogène. Enfin, des choses assez bizarres; enfin, il suffit de pas grand-chose. Il suffit d'un match de foot, on a vu pendant la coupe du monde. Il suffit d'un orage, quelque chose à la télé, ou un événement particulier et donc la fréquentation du magasin change. Donc, il a fallu s'adapter, à cette fréquentation et pour s'y adapter, il a fallu faire des horaires prévus quinze jours à l'avance. [...] Il a fallu passer par un système d'horaires variables. Et pour que les horaires variables fonctionnent, ben il faut diminuer le nombre d'heures par semaine. De manière à gagner en souplesse et pouvoir mettre des plages proches de six heures, donc l'idéal c'est six heures, cinq fois dans la semaine, ça nous fait des contrats de trente heures. [...] Donc il y a eu des contrats 30 heures qui ont été créés. Alors après, on a affiné un petit peu... On a eu les contrats 33 heures, 30 heures, 27 heures, 20 heures, 15 heures, 10 heures. »

$\mathrm{Si}$, dans le discours des distributeurs, le recours au temps partiel est une réponse certes ambivalente mais réelle au problème de la pénibilité, le client constitue manifestement également une justification commode des nouvelles formes d'organisation du travail (LALLEMENT, 1998; JuHLe, 2006). Il semble qu'une approche en termes de mobilisation des travailleurs par le sous-emploi résiste mieux à l'analyse qu'une approche en termes d'adaptation aux contraintes de flux de clientèle. La crainte du chômage d'un côté et la nécessité de s'inscrire durablement dans des carrières professionnelles de l'autre composent une donnée centrale pour la compréhension des modalités de mobilisation de la main-d'œuvre dans la distribution.

\section{Encadré 3}

\section{Être ailleurs au travail}

Même si certaines recherches ont pointé des ajustements locaux dans les formes de mobilisation de la maind'œuvre, c'est la question du sous-emploi qui est surtout mise en avant. Dès le premier jour d'immersion dans un hypermarché parisien en avril 2001, l'enquêteur en observe des indices concrets.

À l'entrée du magasin, une banderole signale aux clients susceptibles de souhaiter passer de l'autre côté du miroir : "Cherche hôtes(esses) de caisse, CDI temps plein ou temps partiel."

Suite à la consultation des données sociales de l'hypermarché, il apparaît que le turnover aux caisses du magasin résulte de l'insatisfaction du personnel (le taux d'insatisfaction est de $100 \%$ pour tous les contrats rompus au cours d'une année) et non d'une mécanique contrôlée par la direction du magasin. Souvent, les jeunes arrivants, comme l'un des cinq collègues de l'enquêteur, mettent fin à leur contrat avant même d'avoir terminé les deux jours de formation.

Paradoxalement, après avoir contribué au démantèlement du noyau dur du collectif par le recours systématique à l'emploi étudiant, le manager des caisses s'efforce aujourd'hui de limiter les dégâts causés par la disparition des anciennes caissières. II affirme consacrer $60 \%$ de son temps de travail à compenser les conséquences d'un fort taux de rotation du personnel : rechercher, auditionner et séduire des candidats à l'embauche, puis prévenir et sanctionner les comportements «déviants " d'un collectif peu investi dans la réussite de l'entreprise.

Depuis cinq ans, certains candidats se réservent des périodes d'essai : ils exigent des contrats à durée déterminée pour ne pas entraver leur mobilité professionnelle. Ils font ainsi preuve d'emblée de leur émancipation par rapport au modèle de mobilisation de la main-d'œuvre en contribuant malgré eux à la fragmentation du marché de l'emploi.

Face au phénomène, le manager des caisses a bien saisi que la sécurité de l'emploi ne constituait pas une incitation suffisante. Pourtant, c'est bien par le statut qu'il tente toujours de retenir les salariés. S'ajoute une nuance importante pour saisir pleinement les évolutions en cours. En les embauchant automatiquement en CDI, le manager ne cherche plus à les «mobiliser", mais à les «fixer» par une contrainte objective : l'impossibilité de toucher les indemnités de chômage suite à un départ volontaire.

Pour autant, les managers s'efforcent de mettre en œuvre des mesures pour permettre une meilleure conciliation entre vie professionnelle et vie familiale des salariés. L'efficacité d'une mesure adoptée en région parisienne, est révélatrice des évolutions subjectives en cours.

Pour résorber l'insatisfaction du personnel, un manager s'est largement appuyé sur un logiciel de planification horaire (Planexa) permettant d'intégrer les contraintes extérieures des employés que les caissiers sont encouragés à faire valoir sans autocensure, ce qui constitue la particularité de l'initiative.

Fait marquant à la lecture des feuilles de disponibilité : ces empêchements, perçus comme légitimes car exprimés sans retenue, ne se limitent plus, comme auparavant, à des engagements domestiques et des études. Les caissiers de ce magasin, dont 50 \% sont des étudiants, font aussi primer sur leur travail d'autres formes de réalisation individuelle : sport, musique, relations amoureuses, etc. 


\section{Ajustements au “hors-travail"}

Le stéréotype, abondamment diffusé, de la caissière en situation systématique de sous-emploi serait sous-tendu par l'hypothèse d'une conformité entre les modalités de mobilisation de la main-d'œuvre et les préoccupations des travailleurs. Les observations réalisées à partir des années 2001 semblent remettre en cause cette adéquation. Le terrain en région parisienne par exemple, soumis à un fort turnover, souligne bien ces limites (voir encadré 3 ). Il montre que les managers doivent aujourd'hui recourir à de nouvelles formes de compensation pour soutenir l'attractivité du secteur et assurer le maintien du personnel en caisse.

Ces ajustements des managers sont autant de preuves de leurs tentatives d'adaptation aux profils de collectifs rajeunis par la présence accrue d'étudiants. Ils concernent par capillarité une population de travailleurs plus large, ce qui invite à s'interroger sur la mise à distance du travail salarié.

\section{Le maintien de la valeur "travail"}

À l'origine des résistances aux anciennes modalités de mobilisation de la main-d'œuvre, ces nouveaux arbitrages n'expriment en réalité pas le rejet de la valeur «travail». L'expérience relatée par un manager du Nord indique que de nombreux jeunes peu impliqués en tant que caissiers (retards fréquents, absentéisme, etc.) se montrent très investis dans d'autres contextes de travail comme celui des stages ou au gré de mutations dans d'autres secteurs du magasin. Il en va de même pour les étudiants qui font primer leur formation sur le travail en caisse, privilégiant leurs stratégies professionnelles à long terme. Enfin, certains caissiers, exprimant une forte mise à distance du travail salarié, sont susceptibles de s'y consacrer pleinement s'ils ont le sentiment de pouvoir s'y réaliser. Ainsi, l'hypothèse d'un rejet de la valeur «travail», fondée sur le postulat d'une frontière infranchissable entre travail salarié et loisir, ne résiste pas aux observations. En réalité, les caissiers les moins engagés professionnellement ne fuient pas tant le monde du travail en général qu'une activité dévalorisée et sans perspective d'évolution.

La mise à distance ne se traduit pas seulement par l'augmentation du turnover. La croissance des attentes des salariés et de leurs besoins hors travail (les études en particulier, mais aussi nombre d'activités contribuant à leur « développement personnel»), conduit également à un désinvestissement subjectif au travail. On enregistre alors, surtout en région parisienne, mais également dans les magasins de notre enquête en province, une augmentation importante des pratiques de résistance passive (absences, retards) et actives (vols, casse, etc.). Elles prennent parfois une importance telle qu'elles obligent les distributeurs à mettre en place des stratégies de compensation (voir encadré 4).

\section{Encadré 4}

\section{Exemples de mesures compensatoires mises en œuvre par un distributeur prenant en compte le "hors-travail" des salariés}

En 2006, l'enquête reprend sur le premier terrain. Le manager des caisses a stabilisé son taux annuel de turnover autour de $50 \%$. II s'est pour cela appliqué à valoriser l'activité et les avantages proposés par son entreprise. II a surtout accompagné les aspirations des employés à se réaliser hors du lieu de travail, en adaptant les horaires à leurs besoins et en facilitant les échanges à l'intérieur du collectif.

Le travail ne permettant pas, au moins à moyen terme, de répondre aux attentes professionnelles des caissiers, ces derniers se sont investis ailleurs... et le manager les a suivis. La gestion a désormais intégré les contraintes familiales, les études et les loisirs en les considérant comme une priorité sur l'activité professionnelle. À titre d'exemple, Nadine, 30 ans, a bénéficié d'arrangements horaires et peut désormais se passer de mode de garde pour ses enfants, ce qui a stabilisé sa situation et mis fin à ses absences répétées. De la même manière, l'allégement des horaires en période de partiels contribue à encourager les étudiants à conserver leur emploi. Tandis qu'auparavant ils quittaient leur employeur au moment des examens, ils tendent aujourd'hui à rester jusqu'à l'obtention de leur diplôme.

Enfin, incités à faire valoir des contraintes élargies qui justifient certaines indisponibilités au travail, d'autres salariés assument donc leur investissement dans des activités situées à l'intersection de la sphère professionnelle et des loisirs: Simon, 19 ans, refuse des heures complémentaires pour préparer son prochain concert dont la maigre recette compensera toutefois le manque à gagner.

Dans l'ensemble pourtant, les compensations sont rarement à la hauteur des besoins réels. Dans la plupart des cas, comme l'observe Nathalie CatTAnÉo (1997), les conditions de travail en caisse mènent encore souvent à des désinvestissements des autres sphères de vie. Selon l'Observatoire de la vie étudiante (OVE), au-delà de vingt heures de travail hebdomadaires (synonymes, dans beaucoup de magasins, de passage aux horaires variables), les étudiants désertent les amphithéâtres, les sportifs sont dans l'incapacité de participer régulièrement aux entraînements et cessent leurs activités physiques.

Comme les horaires flexibles compliquent l'articulation des temps de vie, les caissiers peuvent rapidement s'user d'autant qu'à partir de 30 ans, le sas entre études et travail tend à se fermer et à se vider de sa valeur de parenthèse. Les caissiers cherchent dès lors à concilier durablement et plus harmonieusement le statut de leur emploi, le contenu de leur activité et les revenus de leur travail. Des 
projets professionnels se raniment alors parfois au gré d'une opportunité. C'est le cas de Sophie, 32 ans, revue cinq ans après notre première enquête, comme cliente cette fois dans le même magasin. Conformément à ses souhaits, elle travaillait autrefois sur la base d'un contrat d' «hôtesse de caisse» de vingt heures par semaine, avec des horaires aménagés pour faciliter la garde de ses deux enfants. Quand on lui a proposé un travail proche de ses qualifications dans un syndicat d'initiative, elle a accepté sans hésitation : " J'ai dit oui tout de suite, même à plein-temps. » Certes, elle consacre désormais moins de temps à ses enfants, mais considère avoir avec eux une relation de meilleure qualité : "Et puis c'est mieux de voir moins souvent une mère épanouie, que tout le temps une mère aigrie. »

\section{Des activités "vitales"}

Les arbitrages travail / famille, travail / loisir ou loisir / famille ne sont pas toujours aussi tranchés. Ils ont en commun de mettre en jeu la catégorie d'«activité vitale» que Chantal Nicole-DrancourT (2004) a mise au jour pour la première fois dans une étude des usages sociaux du temps partiel féminin. Selon l'auteure, la notion peut qualifier, comme ici dans un second temps pour Sophie, le travail et la carrière, mais aussi, pour d'autres, le fait de privilégier par moment des activités extraprofessionnelles : l'éducation des enfants (non plus seulement perçue comme une contrainte domestique), le sport, ou même simplement, comme l'a exprimé l'une des caissières, la possibilité de se «retrouver» seule; il s'agit en somme d'activités contribuant à l'épanouissement de soi.

Autrement dit, dans un contexte de travail incitant les individus à se réaliser ailleurs, l'attractivité de l'intégration par le statut tend à décliner au profit d'un intérêt croissant pour la nature même des activités de travail. Les normes - même si les pratiques des caissiers indiquent qu'ils ont tendance à s'en détacher - continuent néanmoins de structurer encore largement la réalité institutionnelle : le salariat à temps plein reste en France le seul mode d'accès à la citoyenneté économique et sociale (Letablier, Nicole-Drancourt, 2007, p. 249), ce qui explique la permanence de la préoccupation pour la stabilité de l'emploi et les ambivalences exprimées par les enquêtés les plus menacés (voir l'exemple de Marianne dans l'encadré 7). Il en résulte un décalage croissant entre les attentes des caissiers et la possibilité de les réaliser. Le phénomène constitue un facteur majeur d'insatisfaction et de résistance au travail.

Ce décalage est important en région parisienne. Il est généralement moins marqué en province, ainsi que le soulignent deux indicateurs : le turnover de l'enseigne (qui correspond uniquement aux ruptures de contrats) est trois fois supérieur en région parisienne à la moyenne des magasins de province
(47\% contre $15 \%$ en 2002 selon les données sociales de l'enseigne). En outre, une étude citée dans le rapport de branche de la FCD en 2004 fait état de difficultés de recrutement en hausse régulière pour l'ensemble des points de vente en France, avec une forte disparité Paris / province. L'«indice de difficulté de recrutement» s'élève à $12 \%$ en région parisienne alors qu'il tombe à $6 \%$ pour les zones les plus concernées du reste de la France.

Les auteurs attribuent cette caractéristique à la concentration d'employeurs situés en Île-de-France. Ainsi, le grand nombre d'offres d'emploi proposées en Île-de-France donne le sentiment qu'il est facile de décrocher un poste, ce qui inviterait, davantage qu'en province, les caissiers au nomadisme professionnel. Outre l'implantation géographique des enseignes, d'autres variations se situent au niveau des collectifs eux-mêmes. Et l'analyse comparée des entretiens et des pratiques révèle par ailleurs des différences significatives du degré d'adhésion aux normes d'emploi en fonction de la qualification et de l'âge. Sans surprise, la qualification incarne un potentiel à réaliser des aspirations de carrière. Les enquêtés les moins qualifiés, susceptibles de bénéficier d'une promotion interne en faisant leurs preuves sur le terrain sont limités à leur entreprise. Ils restent donc plus attachés à leur emploi.

Enfin, plus le salarié est âgé, plus il est probable qu'il se trouve dans un rapport de proximité avec des normes d'emploi marquées par la stabilité dont l'apogée se situe à la fin des Trente Glorieuses. Par ailleurs, augmente avec les années la probabilité d'être confronté à des événements sédentarisant ou nécessitant des ressources plus régulières (installation hors du foyer parental, maternité / paternité, etc.).

\section{Une typologie dynamique du rapport à l'activité et à l'emploi}

Le croisement des différents facteurs dessine la typologie d'un processus : celui d'un investissement identitaire qui passe progressivement du statut à l'activité. Les différentes formes de lien au travail en caisse se présentent sous la forme d'un continuum entre deux pôles. À chacune des extrémités se situent les figures a priori les plus stables : les étudiants et les «reines», c'est-à-dire les plus anciennes. Entre les deux, on trouve une population très hétérogène, souvent précaire, caractérisée par une forte tension entre les attentes et la réalité vécue au travail.

\section{Des étudiants : entre l'“au dehors" et l'“au-delà" du travail en caisse}

Les étudiants sont principalement compris dans une fourchette d'âge allant de 18 à 23 ans et travaillent dix, quinze voire, plus rarement, dix-huit à vingt heures par semaine. Ces jeunes (autant de 
femmes que d'hommes) forment une grande partie de l'effectif des caisses et la majorité des embauches aujourd'hui. Selon les politiques d'emploi des enseignes, ils peuvent représenter de 10 à $50 \%$ du collectif. Du fait qu'ils vivent principalement chez leurs parents, leur dépendance économique au travail est inversement proportionnelle à celle qu'ils entretiennent avec leur famille. Le travail, même intermittent, devient donc un moyen de prendre de la distance avec les contingences familiales. Il incarne un "seuil biographiquement décisif», où le jeune sujet est amené à «construire sa vie dans un espace de contraintes, de tensions, de liens, et de ruptures» (CINGOlani, 2012, p. 93). Dans la majorité des cas, les premiers salaires sont marqués du goût de l'insouciance liée à la découverte de l'autonomie financière, comme le suggère Philippe, 22 ans, étudiant en région parisienne :

«Ça a pas mal changé ma vie, parce que j'avais de l'argent de poche, mais j'en avais pas énormément quand même. C'est vrai que tout à coup, je me suis retrouvé [...] avec un peu plus de trois cents euros, alors que d'habitude j'avais juste... enfin, ça dépendait, mais pas grand-chose... ça a surtout changé pas mal de choses au niveau des sorties, de ma manière de consommer. [...] J'offrais des cadeaux à ma copine, je payais des verres à tout le monde. Bref la vie facile quoi...»

Ce sentiment est d'autant plus partagé lorsque les étudiants ne sont pas contraints de travailler pour subvenir à leurs besoins. Parfois, comme Leila (voir encadré 5), des étudiants plus âgés et d'origine sociale modeste utilisent leur salaire pour payer leur logement et leur nourriture. Ils mènent alors une existence austère mais ne partagent cependant pas le sentiment des caissiers les plus précaires, car ils sont soutenus par la promesse d'un «au-delà» professionnel situé au terme de leur cursus de formation.

Dans l'ensemble, les étudiants forment en caisse une population aux attentes et aux besoins assez homogènes (même les moins insouciants): ils escomptent seulement un bénéfice ponctuel, leur avenir professionnel se jouant dans d'autres sphères.

En région parisienne, les étudiants sont plus mobiles, ce que traduit un taux de turnover plus élevé à moins que l'entreprise ne prévoit des dispositifs qui les incitent à rester. Tel est le cas de Patrick, 24 ans, et de certains de ses collègues retrouvés lors d'un retour sur le premier terrain. Parce que le manager a pris des dispositions pour que travail et loisirs s'accordent et que l'entreprise donne accès aux salariés à des réseaux permettant de pratiquer leurs activités à plusieurs (plusieurs caissiers partagent les mêmes passions), ces derniers se sentent finalement à leur place, du moins provisoirement, en caisse. Dès lors que leur situation ne correspondra plus à cette attente, comme d'autres avant eux, ils n'hésiteront cependant guère à changer de travail.

\section{Encadré 5}

\section{Leila, une étudiante moins insouciante que les autres}

Dans la région de Metz, notre enquête nous a donné l'occasion de rencontrer Leila, 25 ans, préparant une maîtrise de sociologie. Son profil contraste avec celui de ses collègues étudiants. Cinq ans auparavant, la jeune fille a dû quitter le foyer familial (situé à $150 \mathrm{~km}$ ) pour rejoindre les lieux de sa formation. En début de cursus, elle a obtenu une bourse qui, en marge de ses études, lui a permis de pratiquer une activité peu lucrative certes mais plus "gratifiante» et en lien avec ses études. Mais... "à partir du moment où [elle] n'a plus eu sa bourse», elle a dû y renoncer pour un emploi plus lucratif. Leila a alors trouvé dans un hypermarché un contrat étudiant qui lui assure un revenu mensuel de base de 300 euros. Pour financer son mode de vie (même spartiate), elle a dû aligner les heures "complémentaires" et les avenants à son contrat de travail durant les vacances. Au prix de lourds sacrifices (elle n'a quasiment plus de loisirs), Leila est aujourd'hui autonome. Elle a appris à jongler avec différentes ressources et à réduire sa consommation.

Sa vie d'étudiante n'a pas grand-chose en commun avec l'existence insouciante de la majorité des caissiers qui partagent son statut. Aujourd'hui, la seule préoccupation de Leila, à l'heure où Philippe et ses amis s'interrogent sur leur destination nocturne de samedi, c'est de terminer sa maîtrise, et partir enfin à la quête d'un «vrai » travail, pour s'offrir une «vraie» vie :

"C'est difficile d'avoir une vraie vie d'étudiant sans pouvoir aller là où les autres vont. C'est difficile de dire toujours "non, je ne peux pas venir avec vous" parce qu'on n'a pas d'argent. Enfin des choses comme ça. Puis il y a aussi la danse que j'aimais bien. J'aimerais bien pouvoir reprendre des cours. Donc tout ça, c'est aussi des motivations pour sortir de là, [...] en finir avec les études, chercher du travail pour recommencer à vivre un peu en dehors, quoi, en dehors de la fac et du travail... d'avoir une vie un peu plus, comment dire, un peu plus égayée. »

Lorsque l'on quitte la région parisienne, on relève un plus fort attachement à l'emploi, y compris chez les étudiants. En général, ils envisagent de rester en caisse jusqu'à la fin de leurs études. Certains (toutefois minoritaires), voyant arriver la fin de leur formation, tentent de négocier une promotion au sein du magasin parce qu'ils redoutent une intégration difficile dans d'autres secteurs d'activité. Dans la pratique, ces revirements subjectifs (ces salariés passent alors dans la catégorie décrite dans le paragraphe suivant) se traduisent par un investissement supplémentaire au travail. Les caissiers concernés se montrent dès lors plus soucieux d'attirer les faveurs de leurs supérieurs. Mais ces nouveaux desseins se heurtent souvent aux «résistances bien intentionnées » des managers, eux-mêmes pénétrés 
de l'idée que le travail en caisse ne peut constituer qu'une activité secondaire, a fortiori pour des jeunes qualifiés.

\section{Les passagers en transit}

La position intermédiaire entre les étudiants et les plus anciennes caissières est principalement occupée par des femmes. Si l'on y trouve aussi des hommes, ils proviennent de la catégorie précédente et leur entourage les presse de quitter la ligne de caisse.

La forme des contrats de cette catégorie de personnel est rarement celle qu'impose l'organisation du travail. En effet, chaque magasin propose un contrat type ( 26 heures, 27 h 30, 28 heures, 30 heures) que les caissiers négocient ensuite autant à la hausse (au moment de notre enquête cependant, il était rare qu'ils obtiennent un temps plein) qu'à la baisse. Il en résulte une forte hétérogénéité des formes d'emploi et des profils. Dans cette catégorie, les enquêtés ont au moins un point commun: le sentiment d'avoir "fait naufrage» en caisse, après un accident de parcours qui s'est généralement produit au cours de leur scolarité. Les plus qualifiés proviennent souvent de la catégorie précédente (les étudiants) : suite à un échec en cours d'études, ils se sont rabattus sur l'activité exercée en marge de leur formation. Dans certains cas, dominés dans et par le système universitaire, ils finissent par entretenir un rapport ambivalent et mouvant à leur «job étudiant» auquel ils accordent une importance croissante à mesure qu'ils se retrouvent en porte-à-faux dans leur cursus scolaire. Ces modalités d'investissement dans le travail rejoignent ce que certains travaux ont pu relever sur les caractéristiques de l'emploi étudiant dans les fast food (PINTO, 2007 ; CARTRON, 2005).

\section{Encadré 6 \\ Des salariés hors cadres et des managers surpris par le surinvestissement du travail}

Depuis quelques années, le manager des caisses du magasin enquêté en région lilloise a fort à faire avec des étudiants, toujours plus nombreux, qui, à la suite d'un incident en cours d'études, ont choisi les caisses en solution de repli. Plutôt que de prendre appui sur leur engagement au travail, l'homme se fait un point d'honneur de les «remettre sur les rails". Ayant lui-même un parcours un peu atypique, il profite de ses nombreux contacts en dehors de la grande distribution pour leur trouver une porte de sortie honorable. "Caissière, c'est pas une perspective", nous dit-il à propos de MarieLaure, 23 ans, à qui il s'apprête à annoncer une proposition de formation en alternance, dans un autre secteur d'activité.
Dans cette population encore, les caissiers de province se distinguent de ceux de la région parisienne par un investissement plus élevé au travail. Ceux que nous avons rencontrés en Île-de-France ne se sont jamais projetés dans une carrière passant par les caisses. En province, par contre, les salariés tentent parfois d'arrimer leur destin professionnel à la situation plus ou moins stable dont ils pensent bénéficier dans l'hypermarché. La réaction de leurs supérieurs à cet engagement inattendu en dit long sur l'inadéquation des structures à leurs attentes (voir encadré 6).

Quant aux moins qualifiées (toujours des femmes dans notre enquête), elles concentrent davantage leurs attentes professionnelles sur les caisses, ou bien s'en retournent au foyer. Découragées par les échecs scolaires successifs, elles hésitent à se lancer dans de nouvelles formations, seules clés, pourtant, d'un avenir professionnel correspondant à leurs espoirs initiaux. Elles ont donc reformulé leurs exigences pour les adapter à la situation. Au-delà d'une période de mise à l'épreuve assez bien tolérée par les plus jeunes, les caissières peu qualifiées sont attachées à la sécurité de l'emploi (en province surtout). C'est le cas d'Annette, 21 ans, embauchée en contrat de qualification dans la région lilloise. La jeune femme dit avoir apprécié ses premières expériences de courte durée, car elles répondaient alors à une aspiration au changement : "Non, moi j'aime bien bouger, c'est bien de changer, on voit beaucoup de choses. „ Aujourd'hui, Annette revendique le droit à la stabilité, qu'elle considère nécessaire pour envisager une évolution de carrière :

"Non parce que, à 21 ans, quand même, c'est bien d'avoir un travail stable maintenant [...] Tu vois, d'ici, après, peut-être je peux monter; tu vois, si je travaille bien, bon ben le chef il verra.»

Quelques caractéristiques distinguent encore la région parisienne et la province. En région parisienne, ceux qui ont échoué en caisse n'y restent jamais très longtemps. Les anciens étudiants attendent le début d'une prochaine formation (et ne cessent donc pas de se considérer comme étudiants) ou restent à la recherche d'un emploi dans un autre secteur d'activité.

Prises dans les aléas des obligations familiales, les mères (souvent célibataires) doivent parfois mettre en attente leurs ambitions de carrière. Si elles bénéficient de situations favorables comme l'appui d'un conjoint ou l'aide des parents, elles ne tardent pas à chercher des opportunités hors de l'hypermarché. Certaines, comme Sandra, 31 ans, prennent le risque :

«Pour moi j'ai pas peur, il vaut mieux que je parte avant que ce soit trop tard, même si j'ai pas encore trouvé. Comme ça au moins j'aurai la motivation pour chercher. [...] En attendant, avec le salaire de mon mari, et puis les primes, on peut s'arranger. [...] Bon pas longtemps hein, mais au moins pour quelques mois tu vois.» 


\section{Encadré 7 \\ Marianne entre deux feux}

Marianne, Limoges, 29 ans, passe 27 h 30 par semaine en caisse, ce qui lui laisse le temps de s'occuper de son fils de 3 ans. Au moment où l'enquêteur l'a rencontrée, elle est partagée entre travail et famille, entre sécurité de l'emploi et évolution professionnelle.

Arrivée dans la grande distribution après une longue période de chômage, Marianne a d'abord considéré sa situation comme un soulagement provisoire : "en attendant mieux». Avec le temps, elle a commencé à s'y faire, à participer aux réunions du comité d'entreprise; elle est même devenue animatrice d'îlot... au point de s'y sentir à sa place.

Un événement dans la sphère familiale, la naissance de son fils, va pourtant réveiller ses ambitions professionnelles et, simultanément, l'angoisse de ne pouvoir les réaliser :

"Maintenant euh, ben c'est vrai que depuis deux-trois ans, surtout depuis que mon fils est né, ben, j'ai vraiment envie de faire un travail, comment dire, euh, plus valorisant et plus intéressant. »

Elle songe alors à une évolution professionnelle, tiraillée entre aspiration au changement et inquiétude de ne pas trouver ailleurs un emploi stable. Elle se concentre d'abord sur son entreprise puisque son intégration y est assurée. Elle redouble alors d'efforts pour y engranger des expériences susceptibles de soutenir ses projets d'évolution. Seulement, les opportunités manquent...

"Chaque fois qu'il y a une opportunité de remplacer quelqu'un en caisse centrale, j'y vais, je suis à l'affût de tout. Comme ça, quand un poste s'ouvrira, si un jour ça arrive, moi je serai prête, voilà oui. II faut que je sois prête au cas où. »

Aujourd'hui, Marianne hésite encore à prendre la décision qui la conduirait probablement à laisser derrière elle son enseigne et la sécurité de l'emploi. Une chose est toutefois certaine : si elle part, "ce n'est pas pour rester caissière ", dit-elle. Il est grand temps "de faire enfin quelque chose de plus intéressant».

En province, on rencontre surtout des femmes mariées ou vivant en concubinage. En région parisienne, la même situation familiale les aurait probablement encouragées à prendre des risques. À l'inverse, ici, elles finissent souvent par se satisfaire de leur situation. Sédentaires un peu par défaut, elles compensent leur manque d'intérêt à la tâche par un investissement dans le collectif de travail. Elles participent aux sorties organisées par le comité d'entreprise et aux activités syndicales de manière plus intense qu'en région parisienne.

Quant aux caissières les moins jeunes, elles trouvent souvent refuge dans des niches où elles bénéficient d'un peu plus de reconnaissance : elles occupent donc fréquemment les postes de facturation, d'accueil et de caisse centrale, qui offrent une plus grande variété de tâches et de scripts comportementaux que les caisses classiques.

Cette stratégie des plus âgées répond également à la volonté de se soustraire à la monotonie du travail en caisse devenant avec le temps de moins en moins tolérable. Ainsi, la question de la pénibilité - qui ne représente pas vraiment un enjeu pour les plus jeunes et les étudiants - est étroitement associée au facteur âge dans notre typologie. Cela explique en partie qu'à qualification égale, les plus jeunes soient moins sensibles à ces aménagements du quotidien au travail et davantage à l'articulation entre le travail et le hors-travail.

Même si les évolutions se heurtent chez les jeunes caissières à des nécessités contradictoires (voir encadré 7), leurs comportements en province et en région parisienne ont tendance à converger : en témoigne depuis quelques années la baisse de l'écart entre le taux de turnover des magasins de Paris et celui des magasins de province de l'enseigne.

\section{Les « reines"}

Les plus anciennes caissières, parfois nommées les «reines» par leurs collègues, ferment la typologie. Ces femmes sont souvent perçues, et se perçoivent elles-mêmes, comme les vestiges d'un temps révolu. Elles expriment souvent en entretien que ce qui est bon pour elles ne l'est certainement pas pour les plus jeunes. Elles cultivent un rapport au travail salarié spécifique à leur génération (et à leur genre). Elles sont généralement très attachées à leur statut, non pour la sécurité qu'il confère, mais parce qu'il symbolise leur émancipation de la sphère de «reproduction» et ce, dans le cadre plus général d'un rapport positif à l'emploi, tel qu'il a été analysé par Philippe Alonzo (1998). L'exemple de Danièle, 47 ans, dans la région de Metz, en 2002, est emblématique. Elle a toujours travaillé (y compris comme salariée) dans l'ombre de son foyer. Après vingt-cinq ans de bons et loyaux services à la maison, elle a aujourd'hui le sentiment de mériter d'être reconnue pour son travail salarié.

"Ça je veux qu'ils [sa famille] le reconnaissent. Et ils le reconnaissent très bien, donc il n'y a pas trop de souci de ce côté-là. Parce que si tu veux, je veux qu'ils respectent ça. Moi en tant que maman à la maison, en tant que femme, en tant qu'épouse, en tant que mère au foyer qui fait leur popote et tout, et en contrepartie, moi, je travaille aussi donc, tout le monde doit respecter ça et ça se fait très bien. »

Elle a donc payé par son investissement au foyer le «droit» de se consacrer un peu à elle. En l'autorisant à sortir de l'ombre, son mari et ses enfants lui accordent une reconnaissance sociale. Depuis qu'elle a un pied dans l'entreprise, Danièle prend une part active dans les discussions à table en famille (auparavant, lorsqu'elle était assistante maternelle, personne ne prêtait attention, dit-elle, à ses «pipis cacas popos»). Elle peut désormais partager aussi ses expériences avec des collègues de travail. 
Parce qu'il leur confère une reconnaissance dans la sphère publique, les anciennes caissières expriment un attachement marqué à leur travail. Même si la pénibilité peut contribuer à limiter leur investissement, ce sont généralement des employées disponibles (leurs enfants sont grands) et attentives aux besoins des clients... Mais sur le point de disparaître des hypermarchés. La génération des «reines» a tracé le chemin pour les suivantes : désormais, les jeunes femmes ne doutent plus de la légitimité de leurs ambitions professionnelles. Elles ont par contre encore des craintes sur leur faisabilité, en particulier quand il faut les concilier avec les exigences de la maternité. C'est pourquoi, certaines d'entre elles restent très attachées à la sécurité de l'emploi. Quant aux jeunes caissières, elles s'inquiètent davantage encore d'être enfermées dans un secteur d'emploi sans perspective de carrière et de plus en plus éloigné de leurs attentes professionnelles.

$*$
$*$
En insistant sur les retournements biographiques
qui changent le rapport au travail, à l'emploi et à

En insistant sur les retournements biographiques
qui changent le rapport au travail, à l'emploi et à la vie privée, la typologie issue du croisement de trois facteurs discriminants (lieu, âge et qualification) met en valeur l'hétérogénéité des manières d'être au travail. Issue d'une enquête de terrain, cette typologie nous permet d'éviter d'établir un lien automatique entre le statut d'emploi et le vécu subjectif. Elle invite plutôt, notamment à travers le cas des étudiants en caisse, à suivre les modulations des expériences subjectives vécues dans une situation professionnelle donnée. Ainsi avons-nous pu dévoiler différentes figures dissimulées derrière l'habituel portrait de «la caissière» en hypermarché : nous avons montré que la précarité, souvent associée comme par réflexe au travail en caisse, ne se réduit pas à un état objectif, mais se situe à la rencontre d'une trajectoire et d'une réalité de travail et d'emploi. Enfin, en s'écartant d'une interprétation systématiquement négative de la discontinuité professionnelle, notre analyse souligne l'enjeu subjectif croissant constitué par l'appropriation du temps et la fragilité des articulations entre le travail et le hors-travail. Comme c'est le cas dans la grande distribution, cette évolution est susceptible de produire certains désajustements avec les politiques de mobilisation de la main-d'œuvre et les représentations de l'emploi portées au sein des organisations.

\section{Bibliographie}

Alonzo P. (1998), «Les rapports au travail et à l'emploi des caissières dans la grande distribution. Des petites stratégies pour une grande vertu», Travail et emploi, $\mathrm{n}^{\circ} 76$, pp. 37-51.

Angeloff T. (2000), Le temps partiel: un marché de dupes?, Paris, Syros.

Appay B. (2005), La dictature du succès : le paradoxe de l'autonomie contrôlée et de la précarisation, Paris, L'Harmattan.

BA A., Vignon C. (2013), «Mieux gérer les incidences de l'automatisation des services : le cas des caisses libreservice», Gestion, vol. 38, n² 2, pp. 62-70.

Battagliola F. (2004), Histoire du travail des femmes, Paris, La Découverte.

Beau A.-S. (2004), Un siècle d'emplois précaires, Paris, Payot.

Benquet M. (2011), Les damnées de la caisse : enquête sur une grève dans un hypermarché, Bellecombe-enBauges, Éditions du Croquant.

BERnARD S. (2012a), «La promotion interne dans la grande distribution: la fin d'un mythe?», Revue française de sociologie, vol. 53, $\mathrm{n}^{\circ} 2$, pp. 259-291.

Bernard S. (2012b), «Conflits de temporalités dans les services. Le cas des caisses automatiques », Temporalités, $\mathrm{n}^{\circ} 16$; disponible en ligne à l'adresse : http://temporalites. revues.org/2250; consulté le 14 décembre 2013.

Bernard S., Chappaz F. (2007), «Hétérogénéité de l'emploi et compromis temporel : les emplois étudiants », in Vatin F. (dir.), Le salariat : histoire, théorie et formes, Paris, La Dispute, pp. 261-278.

Boitel L., Desarmenien A., Dupéry M., Gaucher M., Incorvaia A.-M., Jeancolas B., Pommier J.-L., Rat De Coquard M., Viossat M., Ergodistrib (2007), Rapport interne. Résultat de l'enquête santé et de l'étude ergonomique des postes de travail. La santé à tous les rayons, Paris, Docis.

Bouffartigue P., Pendariès J.-R. (1994), «Formes particulières d'emploi et gestion d'une main-d'œuvre féminine peu qualifiée: le cas des caissières d'un hypermarché», Sociologie du travail, vol. 36, $\mathrm{n}^{\circ}$ 3, pp. 337-359.

BuÉ J., Rey F., Roux-Rossi D. (2013), «Le temps partiel moins attractif? Nouvelles régulations temporelles dans deux secteurs de services», Travail et emploi, $\mathrm{n}^{\circ} 134$, pp. 59-74.

Cartron D. (2005), «Engagement dans le travail et dans la grève chez McDonald's», in Denis J.-M. (dir.), Le conflit en grève?, Paris, La Dispute, pp. 251-268. 
CATtANÉo N. (1997), «Précarités et travail à temps partiel des femmes. L'exemple des caissières d'hypermarché», in Appay B., Thébaud-Mony A., Précarisation sociale, travail et santé, Paris, Institut de recherche sur les sociétés contemporaines, pp. 331-340.

Chaney C., Alcouffe J., Frau-Prudhomot P., Le Pache J., Manillier P., Montéléon P.-Y., Mora V., SAnchezBRÉCHOT M.-L. (2011), «Vieillissement et travail dans la grande distribution alimentaire», Camip.Info. L'actualité de la santé au travail, $\mathrm{n}^{\circ}$ 2011-1.

Cingolani P. (2012), Le temps fractionné : multi-activité et création de soi, Paris, Armand Colin.

Dubar C. (2000), La crise des identités : l'interprétation d'une mutation, Paris, Presses universitaires de France.

DurAnd J.-P. (2004), La chaîne invisible. Travailler aujourd'hui: flux tendu et servitude volontaire, Paris, Seuil.

Garabuau I. (1997), "Cols croisés, cols droits et tee-shirts. Étude de classifications chez des caissières de supermarché», Anthroepotes, vol. II, n 4, pp. 2-21.

JuHLE S. (2006), «Les employés de la grande distribution : entre le chef et le client», Travail et emploi, $\mathrm{n}^{\circ} 105$, pp. 7-18.

LALlement M. (1998), «Relations professionnelles et emploi : du niveau à la configuration», Sociologie $d u$ travail, vol. 40, n² 2 pp. 209-231.

Le Corre S. (2001), «Transformation des marchés du travail et perspectives d'analyse : réflexion à partir du cas de la grande distribution alimentaire», Actes des VII journées de sociologie du travail. Marchés du travail et différenciations sociales, pp. 139-147.

Le Corre S. (1991), «Modèles d'entreprises et formes de gestion sociale dans les hypermarchés : diagnostic et évolution », Formation emploi, n 35, pp. 14-25.

Letablier M.-T., Nicole-Drancourt C. (2007), «Le salariat féminin et le modèle de l'intermittence», in Vatin F. (dir.), Le salariat : théorie, histoire et formes, Paris, La Dispute, pp. 243-260.

Mолті P. (2001), L'avenir de la grande distribution, Paris, Odile Jacob.

Nicole-Drancourt C. (2004), «Les usages sociaux de l'emploi à temps partiel dans les trajectoires de vie contemporaines ", in Collectif Griot, Figures du temps : les nouvelles temporalités du travail et de la formation, Paris, Budapest, Torino, L'Harmattan, coll. «Logiques sociales. Cahiers du Griot», n 1, pp. 65-77.

Pillon T. (2007), «Les intérimaires et le temps», in Vatin F. (dir.), Le salariat : théorie, histoire et formes, Paris, la Dispute, pp. 279-297.

Pinto V. (2007), " Servir au fast food. "Petit boulot" et engagement dans le travail», Revue Agone, n 37 ; disponible en ligne à l'adresse : http://revueagone.revues. org/706; consulté le 14 décembre 2013.

Prunier-Poulmaire S. (2000), «Flexibilité assistée par ordinateur : les caissières d'hypermarché», Actes de la recherche en sciences sociales, $\mathrm{n}^{\circ} 134$, pp. 29-36. 